\section{Monkeys bridge the stroke gap}

\section{By Michael J. Haas, Senior Writer}

Toronto-based NoNO Inc. has become the first company to show that macaque models have the potential to derisk clinical testing of new stroke therapies, which typically have gone right from rat or rabbit models into humans. In February, the company reported that its neuroprotective peptide NA-1 was effective both in macaque models of stroke ${ }^{1}$ and in a Phase II trial to treat ruptured brain aneurysms.

The only drug in the U.S. to treat ischemic stroke is the clot buster Activase alteplase from Roche's Genentech Inc. unit and Boehringer Ingelheim $\mathbf{G m b H}$, which was approved in 1996. Since then, numerous neuroprotective therapies have shown efficacy in standard rat models of stroke, but all have failed in the clinic.

In 1999, the Stroke Academic Industry Roundtable (STAIR) recommended testing neuroprotective agents in nonhuman primate models of stroke because their brains are anatomically and biologically similar to humans, and they can be given neurological and behavioral tests similar to those used to measure outcomes in clinical trials. ${ }^{2}$

But the specialized facilities, expertise and costs associated with such models have largely precluded their use, said Michael Tymianski, cofounder, president and CEO of NoNO. He is also assistant professor of surgery and physiology at the University of Toronto, staff neurosurgeon at Toronto Western Hospital and senior scientist at the hospital's Toronto Western Research Institute.

Previous Tymianski-led teams showed the neuroprotective effects of NoNO's NA-1 (Tat-NR2B9c) in rat models of ischemic stroke. ${ }^{3,4}$ The compound inhibits discs large homolog 4 (DLG4; PSD95). NA-1 is a 20-mer peptide composed of the 9 C-terminal amino acids of NMDA receptor NR2B subtype (GRIN2B; NR2B) linked to an 11-mer HIV Tat peptide that allows NA-1 to cross the blood brain barrier.

In its latest study, Tymianski's team developed two macaque models of surgery-induced stroke and tested NA-1 in the animals. The models involved clamping off the middle cerebral artery to induce ischemia, administering NA-1 and then removing the clamp to allow reperfusion of the ischemic zone.

The two models differed in the placement of the clip to induce either rapid-onset strokes that are more severe than those usually seen in humans or slowly evolving strokes that resemble those frequently presented by patients. The team reasoned that showing a therapeutic effect for NA-1 in both models would increase the chance of eventual success in the clinic, Tymianski said.

Macaque models given NA-1 1 hour after the onset of severe stroke and up to 3 hours after the onset of slow-evolving stroke had smaller infarct sizes 48 hours after reperfusion than animals given placebo. The treated animals also had better neurobehavioral and sensorimotor performance on day 14 than untreated animals. Data were reported in Nature. ${ }^{1}$

Also last month, at the International Stroke Conference, NoNO presented results from a Phase II trial of NA-1 to treat subarachnoid hemorrhage (ruptured brain aneurysm) showing that NA-1 lowered ischemic brain damage and led to better neurological scores on day 30 than placebo.

"NA-1 is the first neuroprotective agent for stroke that, after testing in macaque models, went on to show benefit in a Phase II trial," Tymianski said. "By showing preclinical proof of concept in macaques, we potentially derisked the human experiment" and made a case for wider use of macaque models in preclinical testing of such therapies. "Without the macaque data, we would have been making the leap from rodents to humans-a leap that has failed with every previous therapy."

"The data presented in the Nature study and the data from NoNO's Phase II trial represent a significant and much-needed step forward" in stroke research, said Thomas Sager, head of the Department of Neurodegeneration at H. Lundbeck A/S. "NoNO's approach bridged the gap between preclinical and clinic studies, thereby increasing the likelihood of seeing a therapeutic effect in humans. We need more experiences and papers like this to put new energy into the field of stroke and stimulate a new way of thinking about how to treat it."

Lundbeck has two stroke therapies in clinical development. Desmoteplase (DPSA), a genetically engineered salivary plasminogen activator from the vampire bat Desmodus rotundus, is in Phase III testing. Lu AA24493, a carbamylated form of human erythropoietin (EPO), is in Phase I testing. Lundbeck is co-developing the therapies with Bayer AG and Warren Pharmaceuticals Inc., respectively.

Lundbeck obtained desmoteplase in 2005 from Paion AG, which in turn in-licensed the compound from Schering AG, now part of Bayer.

Victor Gurewich, cofounder and scientific director of Thrombolytic Science International LLC, agreed with Sager. "This is an impressive paper that should interest all of us in the stroke community because it is one of the few studies showing success for neuroprotection in monkeys," he said.

Gurewich is also professor of medicine at Harvard Medical School and director of Beth Israel Deaconess Medical Center's Vascular Research Laboratory. 
Thrombolytic Science's TS01, a combination of a recombinant form of pro-urokinase with one amino acid mutation administered with a plasma complement 1 esterase inhibitor, is in preclinical development to treat stroke. The company expects to begin Phase I testing this year.

\section{Segment segue}

"We need more experiences and papers like this to put new energy into the field of stroke and stimulate a new way of thinking about how to treat it."

-Thomas Sager, H. Lundbeck A/S
Sager said NoNO's preclinical data also point to the need to segment stroke populations.

"Stroke is a complex disease that occurs in a heterogeneous population with many factors of comorbidity," Sager said. "There are many preclinical models of stroke, but at best, only a few reflect the real-life aspects of the disease and none mimics what happens to stroke patients at large."

He added that NoNO circumvented this problem by "testing its therapy in animals that model the clinical situation of one well-defined segment of the stroke population-patients undergoing endovascular repair of intracranial aneurysms." Then, instead of attempting to translate those findings to the general stroke population, the company translated them into a small segment of stroke patients who have an experience very similar to the macaque models, he said. "I think this approach of segmenting the stroke population and developing models of those segments - whether in rats or macaques-points to a new standard for preclinical studies."

The upshot, said Sager, is that the stroke field likely requires a "matrix of complementary stroke models that represent different aspects of the disease and subgroups of stroke patients."

Indeed, there was no clear consensus on whether NoNO's macaque models could have broader applications in the development of stroke therapies.

Gurewich suggested using the team's macaque models to test neuroprotective agents that previously succeeded in rat models but failed in humans. "If those therapies also failed in the macaques, that would further strengthen the argument for using macaques as a bridge between rat models and clinical trials," he said.

Tymianski agreed that testing failed neuroprotective therapies in the macaque models could "clinch the notion that macaques may be an essential translational step" for such therapies. However, he noted, "It is our view that primates should be used for translational studies only if there is a clear commitment to take a successful candidate into human testing."

Sager added: "We also have to be careful not to think about these macaques as models of stroke in general, and instead keep in mind which segment of the patient population this model best represents." Thus, testing therapies that failed to treat a general stroke population in NoNO's macaque models would probably not be meaningful, he said.

Sager said he wants to see macaques and/or other stroke models extended to include aged animals, because "the human stroke population consists largely of older people with diabetes, hypertension and other comorbidities. While it would not be easy to do, age is the simplest aspect of this population to model."
Gurewich was interested in the development of a macaque model of thrombus-induced stroke-in addition to the surgery-induced one NoNO developed.

"For the purpose of demonstrating preclinical proof of concept for NA-1, it doesn't matter whether ischemia occurs as a result of a clamp or a clot," he said. But in a model of thrombus-induced stroke, "you could test the combination of a thrombolytic agent, such as our TS01, and a neuroprotective agent such as NA-1" in a way that reflected how those agents would be used in the clinic.

Tymianski said his team does not plan to develop a macaque model of thrombus-induced stroke. "We have attempted this already, but the variability in infarct size from one animal to the next with this model was too large," he said. "Consequently, the experiments would require population sizes that are not realistic" for nonhuman primate research.

He declined to disclose details about the Phase II trial of NA-1 because the results will be released in a forthcoming publication. $\mathrm{He}$ said NoNO also plans to conduct additional clinical trials of NA-1 to treat subarachnoid hemorrhage and acute ischemic stroke.

NoNO's IP portfolio includes more than 50 patent applications or issued patents covering NA-1 and its use to treat stroke, traumatic brain injury, epilepsy, pain, anxiety and other indications, Tymianski said.

The company has also filed patent applications covering several macaque models of stroke for drug screening purposes. Those models are available for licensing or partnering, Tymianski said.

NA-1 has Fast Track designation from the FDA to reduce procedurally induced stroke and cognitive impairment in patients undergoing endovascular repair of brain aneurysms.

Haas, M.J. SciBX 5(12); doi:10.1038/scibx.2012.301

Published online March 22, 2012

\section{REFERENCES}

1. Cook, D.J. et al. Nature; published online Feb. 29, 2012; doi:10.1038/nature 10841

Contact: Michael Tymianski, Toronto Western Research Institute, Toronto, Ontario, Canada e-mail: mike.tymianski@uhn.ca

2. Fisher, M. et al. Stroke 40, 2244-2250 (2009)

3. Aarts, M. et al. Science $298,846-850$ (2002)

4. Bråtane, B.T. et al. Stroke 42, 3265-3270 (2011)

\section{COMPANIES AND INSTITUTIONS MENTIONED}

Bayer AG (Xetra:BAYN), Leverkusen, Germany Beth Israel Deaconess Medical Center, Boston, Mass.

Boehringer Ingelheim GmbH, Ingelheim, Germany

Genentech Inc., South San Francisco, Calif.

Harvard Medical School, Boston, Mass.

H. Lundbeck A/S (CSE:LUN), Copenhagen, Denmark

NoNO Inc., Toronto, Ontario, Canada

Paion AG (Xetra:PA8), Aachen, Germany

Roche (SIX:ROG; OTCQX:RHHBY), Basel, Switzerland

Thrombolytic Science International LLC, Cambridge, Mass.

Toronto Western Hospital, Toronto, Ontario, Canada

Toronto Western Research Institute, Toronto, Ontario, Canada

University of Toronto, Toronto, Ontario, Canada

Warren Pharmaceuticals Inc., Westchester, N.Y. 